OPEN ACCESS

Edited by:

Arindam Dey,

The University of

Queensland, Australia

Reviewed by:

Sungchul Jung,

Human Interface Technology Lab New

Zealand (HIT Lab NZ), New Zealand

Mark Billinghurst

University of South Australia, Australia

*Correspondence:

Daniel Pimentel

pimend@uoregon.edu

tPresent address: Shiva Halan,

Facebook, Menlo Park, CA, United States

Specialty section: This article was submitted to

Technologies for VR

a section of the journal

Frontiers in Virtual Reality

Received: 19 December 2020 Accepted: 22 March 2021 Published: 23 April 2021

Citation:

Pimentel D, Kalyanaraman S, Fillingim $R$ and Halan $S$ (2021) The Effects of VR Use on Pain Experienced

During a Tattoo Procedure: A Pilot

Study. Front. Virtual Real. 2:643938. doi: 10.3389/frvir.2021.643938

\section{The Effects of VR Use on Pain Experienced During a Tattoo Procedure: A Pilot Study}

\author{
Daniel Pimentel $^{1 *}$, Sri Kalyanaraman ${ }^{2}$, Roger Fillingim ${ }^{3}$ and Shiva Halan ${ }^{2 \dagger}$ \\ ${ }^{1}$ Oregon Reality Lab, School of Journalism and Communication, University of Oregon, Portland, OR, United States, ${ }^{2}$ Media \\ Effects \& Technology Lab, School of Journalism and Communications, University of Florida, Gainesville, FL, United States, \\ ${ }^{3}$ Pain Research and Intervention Center of Excellence, College of Dentistry, University of Florida, Gainesville, FL, United States
}

One of the most socially impactful applications of virtual reality (VR) is its use as a non-pharmacological remedy for both acute and chronic pain. Yet, despite robust findings establishing the analgesic effects of VR, use cases almost exclusively involve (a) patients with acute/chronic pain, which are often difficult to access and vary widely in terms of pain location/severity, or (b) experimentally induced pain, which can have low lab-to-life generalizability. One understudied pain context that may reconcile these limitations is body modification, specifically tattoo procedures. Examining the use of VR during a tattoo offers several benefits to VR and pain research. First, tattoo recipients as a participant pool are more accessible. Second, tattoo pain is presumably more standardized and uniform as it is administered by a machine at a consistent force. Thus, to test these assumptions and expand the scope of VR applications in this domain, we present a mixed-methods investigation testing the effects of VR on pain experienced during a tattoo. Leveraging qualitative interviews with tattoo artists and customers, a 3-month on-site field experiment at a tattoo parlor was conducted. Customers' self-reported pain ratings $(N=16)$ were collected during 1-h tattooing sessions and compared between a treatment (VR) and control group. As expected, VR significantly reduced pain ratings during the procedure, and increased pain resilience. By suggesting that the analgesic effects of VR extend to volitional pain during a tattoo, we argue that tattoo pain warrants attention by both VR content developers and researchers interested in studying how immersive content influences real-world pain perception. The study also yields specific guidelines to help designers create and deploy VR experiences for this context. Overall, the results suggest that tattoo sessions present a promising context worthy of further investigation across a variety of VR research programs.

Keywords: virtual reality, pain, tattoo, body modification, analgesia

\section{INTRODUCTION}

Growing reliance on pharmaceuticals for pain management has contributed to an opioid addiction epidemic that continues to worsen (Lyapustina and Alexander, 2015). In response, leading scholars and practitioners have sought, and identified, various non-pharmacological alternatives (Kolodny and Frieden, 2017). Chief among them is virtual reality (VR), which has served as an effective 
solution for pain management across a multitude of chronic and acute conditions (Mahrer and Gold, 2009; Spiegel, 2020; Trost et al., 2021). By replacing the real-world sensory information (i.e., audiovisual) with computer-generated information in the head-mounted display (HMD), VR experiences elicit a sense of being in the virtual rather than physical world. This sense of "presence" impedes the cognitive ability to process pain signals generated in the real world (Hoffman et al., 2008; Li et al., 2011; Gupta et al., 2018; Scheffler et al., 2018). It is because of this that VR has long been considered "a promising non-pharmacologic analgesic, especially for patients who must undergo brief painful procedures" (see Hoffman et al., 2004, p. 167).

Despite VR's potential analgesic benefits, the efficacy of distraction-based solutions like VR is hindered by several methodological limitations, namely accessibility to patients, as well as low generalizability and internal validity. Moreover, researchers of VR analgesia assert that further research is required on different forms of pain beyond chronic and injury-related pain (Ahmadpour et al., 2019). To address these points and determine the feasibility of VR use in new pain-related contexts, we present a pilot study testing a VR-based pain intervention on a specific population: individuals receiving a tattoo, one of the few contexts wherein users voluntarily seek out a highly painful experience. We outline how testing VR pain interventions on this population can be advantageous to both VR and pain researchers seeking to understand how immersive experiences shape pain perception, albeit a specific form of pain (volitional). Additionally, this work seeks to determine unique user experience considerations to help inform future design and deployment of VR experiences in this unique context.

\section{Methodological Concerns: VR Use During Pain}

VR-based pain research, and by extension the validity and generalizability of its scholarship, is inextricably bound to the characteristics of the subjects it seeks to examine. Dozens of published studies investigating the analgesic effects of VR do so by testing interventions on human subjects with existing medical conditions (e.g., burn victims) (Indovina et al., 2018). While done to ensure a representative sample and ecological validity, the use of such subjects proves problematic for various reasons. Medical conditions, such as burns and chronic back pain, are outside of the control of the subjects and researchers. Furthermore, because each condition varies in severity, it is difficult to draw conclusions from such investigations (Weiss, 1994).

Limiting variability in subjects' pain experience is a noted limitation in most clinical studies using VR interventions (Mahrer and Gold, 2009; Gupta et al., 2018). For example, various studies examining the effects of VR on procedural pain in burn victims face difficulty controlling for the severity and location of the injury (Hoffman et al., 2008; Tashjian et al., 2017), an important factor considering that different regions of the body having varied pain thresholds (Fischer, 1987). Other studies altogether compare different types of injuries (e.g., gunshot wounds, bone fractures) within the same subject pool (Patterson et al., 2010). Another limitation relates to the use of vulnerable subject pools; limited accessibility to participants produces small sample sizes (Gupta et al., 2018). In turn, this limits the ability to control for factors inherent in each form of pain being studied (Dascal et al., 2017), such as uniformity in the pain type/location across participants.

To avoid sacrificing statistical power at the altar of generalizability, studies often place healthy subjects under experimentally-induced pain (e.g., placing hand in ice water) (Law et al., 2010). However, experimental pain has been increasingly scrutinized (Uman et al., 2006), in part due to its weak lab-to-life generalizability (Gerin et al., 2000) and the inability to compare with visceral or chronic pain (Kenny, 2009). Lastly, the experimental designs employed by VR-based pain studies make it difficult to ascertain what psychological factors truly drive the analgesic effects of immersive content (Phelan et al., 2018). Experiments testing the efficacy of VR as a pain reliever primarily do so by comparing a control condition with a VR condition (Schmitt et al., 2011; Kipping et al., 2012; Tashjian et al., 2017). Such modality comparisons, while useful for discerning the platform's effectiveness compared to traditional treatments, fail to advance theoretical understanding of the unique affordances of VR. It is important to note that, like the aforementioned literature, this study does not compare multiple VR conditions. However, a pre-requisite to this methodological approach involves first establishing the viability of VR use during a tattoo procedure and determining its effects on pain perception.

\section{Alternative Contexts for Testing VR-based Pain Interventions Volitional Pain}

This paper proposes a novel methodological approach to more effectively examine the efficacy of VR interventions on pain; one which examines volitional, rather than medical (acute or chronic) or experimentally induced pain. Volitional pain is any form of pain precipitated by a voluntary act (Davies, 2006). Specifically, there is one particular form of volitional pain that is considered painful, commonplace, regulated, and ethically performed: body modification. Body modification is defined as the active, and often painful, process of changing the natural state of one's body (e.g., piercings) (Sweetman, 1999). This study argues that pain associated with volitional body modification, namely receiving a tattoo, is an effective alternative to medical or experimentally induced pain. Compared with aforementioned forms of pain studied in extant VR/HCI literature, volitional pain associated with receiving a tattoo provides unique affordances that make it advantageous from a methodological standpoint.

\section{Tattoo Pain: Methodological Advantages}

First, unlike other forms of pain studied in extant literature (e.g., burns, back pain), the pain-inducing agent responsible for tattoo pain (i.e., the tattoo machine) exerts force onto the skin in a uniform fashion across subjects. That is, where burns may vary in severity and placement, tattoo machines offer stable, and consistently painful, sensory inputs. This is because the tattoo machine injects ink into the skin with a pre-determined speed and force. This reduction in (external) pain variability allows researchers to better isolate the effects of VR-based interventions. 
Second, tattoo procedures, which can inflict prolonged pain for several hours at a time, provide the unique opportunity for the collection of within-subjects longitudinal pain data. This affordance contrasts traditional VR interventions for medical pain which are tested across short time intervals (few minutes) (Hoffman et al., 2000) or no more than 20 min (Hoffman et al., 2014). This limits a study's scope to episodic pain, when in fact pain experiences persist for longer periods. Because of this, pain ratings are collected after, rather than during, the intervention, inhibiting the analysis of factors within VR that shape the pain experience as it occurs. In sum, access to within-subjects longitudinal data allows for the examination of other factors responsible for VR-based pain relief beyond distraction. Third, the growth in popularity of tattoos presents a methodological advantage in terms of sample size and statistical power. This exponential growth of the tattoo industry in the U.S. (Breuner and Levine, 2017), where roughly $30 \%$ of US adults have a tattoo (Beasley, 2011), affords researchers greater accessibility to subjects willingly undergoing a prolonged pain experience.

Considering the aforementioned justification of tattoo pain as a viable methodological substitute for other, more experimentally variant forms of pain, the fundamental prediction that we test in this study is that VR use will reduce perceived pain during a tattoo. To test this hypothesis and lend credence to the use of tattoo recipients as a subject pool with which to test the efficacy of VR-based pain interventions, we present findings from a mixedmethods field experiment. Informed by qualitative interviews with tattoo artists and customers at a local tattoo shop, the experiment tests the effects of VR on perceived pain among individuals receiving a tattoo.

\section{Methodological Overview}

A mixed-methods approach was deemed most appropriate given the interdisciplinary nature of this investigation and topic. That is, use of qualitative and quantitative methods ensured a nuanced perspective on both (a) the phenomenological aspects of administering and receiving a tattoo, and (b) the measurable effects of VR use within this context, respectively. As such, the methodologies used herein relied upon varying disciplinary perspectives and research tactics. Considering standard tattooing procedures and potential safety hazards, establishing a safe experimental procedure by which to test VR simulations on the subject pool was a priority. To the best of our knowledge, no previous study has tested immersive media equipment on participants while receiving a tattoo. Thus, prior to testing any simulation's impact on pain perception during a tattoo procedure, the researchers first attempted to establish selection criteria for a VR simulation to be used in the subsequent field experiment. In this way, the collective inquiry would take into account the entirety of factors unique to a tattoo experience.

A two-phase approach, or a sequential explanatory mixedmethods design, was chosen given the novelty of the context. In the first phase, a researcher conducted semi-structured interviews with experts in the field of body modification, namely tattoo artists at the largest tattoo shop in North Central Florida, as well as customers at the establishment who were both receiving tattoos and inquiring about them.
Furthermore, field observations of several tattoo sessions were also conducted. Artists were shadowed by the same researcher during several procedures, during which they logged field notes. As previously mentioned, both the interviews and observations would allow us to identify factors to be considered when devising the experimental procedures, including what kind of simulation could be used during a tattoo. The qualitative findings subsequently informed phase 2: a 2-condition betweensubjects experiment comparing a VR simulation, selected with consideration of the qualitative findings, with a control group of customers.

\section{QUALITATIVE METHODOLOGY}

\section{Semi-Structured Interviews}

The semi-structured interviews were conducted on-site at a large tattoo parlor in Florida. In total, six (6) tattoo artists (denoted by "A") and two (2) customers (denoted by "C") with preexisting tattoos were interviewed over the course of a week (2018). All interviewees were informed that the researchers were studying how media influences pain experiences, namely tattoos, and willingly agreed to share their experiences and thoughts without financial incentive. The primary purpose of the interviews was three-fold. First, from a practical perspective, the interviews and observations allowed the researcher to familiarize himself with the tattoo experience from both the artist and customer perspective. Artists and customers were asked open-ended questions about their existing tattoos, including general thoughts about the tattoo experience. This included preparation and feelings in anticipation of the tattoo, the steps involved during each procedure, and what occurs afterwards. Furthermore, questions inquired about various tattoo types, areas of the body commonly tattooed, which areas of the body are associated with greater pain sensitivity, and other factors related to the tattoo experience (e.g., cost, body position, duration). To standardize the experimental procedures, it was imperative to establish a sense of how tattoos are administered, and what can be expected from customers and artists, such as the need for breaks, dialogue between artist and customer, and differences in equipment, technique, and style among the artists.

Second, in a related vein, interviews functioned as a feasibility analysis wherein artists and customers provided their thoughts and perceptions about the use of immersive (e.g., VR) and nonimmersive media content (e.g., smartphones) during prolonged pain experiences (i.e., tattooing). These questions provided insight as to whether VR as a media platform could be seamlessly integrated into a typical tattoo procedure.

Lastly, from a phenomenological perspective, interview questions addressed how individuals experience physical pain during a tattoo, its meaning (both the pain and the tattoo), and what factors influence either of the two. These accounts were combined with customer experiences to provide a more systematic and exhaustive understanding of how certain factors of a tattoo (e.g., size, location) could hinder comfortable and safe use of a VR HMD. For example, customers with existing tattoos were asked about their tattoos, the amount of pain they recall during the process, how they were positioned during each tattoo, 
and whether they used any media (e.g., social media apps) during the procedure. Similarly, customers there to receive a tattoo at the time of the interviews were also asked to describe the anticipatory severity (or sensitivity) of the area they were getting tattooed.

\section{Field Observations}

Interview insights were further bolstered by field observations, which were acquired by shadowing the primary tattoo artist onsite for several sessions. During these observational sessions, the researcher logged logistical information about the tattoo experience, including general procedures, time between certain steps, and others. Additionally, the researcher noted any discourse between the customer, the artist, and those in their vicinity. Because the room in which the tattoos were administered was a shared space, with three (3) other stations, notes were also taken as it related to inter-station interactions during data collection.

All conversations were logged via field notes taken at the time of the interviews. Notes were then analyzed and condensed into key insights using an open-coding process that yielded several recurring themes. This yielded rich insights into the entirety of the tattoo experience, including a detailed timeline of the tattoo procedure, a taxonomy of pain sensitivity by body region, and the type of media used by customers during procedures. These insights ultimately helped shape the experimental methodology implemented herein, from stimulus selection to experimental procedures. While not a conflict of interest, it should be noted that the researcher tasked with logging and interpreting the qualitative data has several tattoos, some of which were done at the tattoo shop where the study took place.

\section{Qualitative Results}

\section{Tattoo Timeline}

Findings revealed tattoo procedures exhibited a consistent structure. For multi-hour tattoos, the first hour of the procedure was broken down into three segments: monitoring, tattooing, and break. During the monitoring period (first $15 \mathrm{~min}$ ), the tattoo artist monitors the customer's reaction to the tattoo pain. If a customer exhibit signs of extreme distress, or becomes non-responsive, artists stop the tattoo immediately and either offer the customer water/food or reschedule the session entirely. Should the customer exhibit no signs of an adverse reaction to pain beyond what is deemed normal, the tattooing proceeds uninterrupted for the remaining $45 \mathrm{~min}$ or so. After the initial hour, the artist and customer both take a 10- to 15-min break, repeating this cycle until the tattoo is finished. A specific insight uncovered by the qualitative data found that the tattooing procedure is considered more painful when tattooing commences immediately after a break. A1 stated that "The worst pain is right after a break due to open flesh." Additionally, C1 stated, "I just wanted to get it over with, but I had to get breaks because of the artist."

Qualitative data thus suggests that tattoo procedures offer a 1 - $\mathrm{h}$ window where media use would be acceptable, and pain would be generally consistent in terms of severity. Limiting data collection to the initial hour also addresses factors associated with physiological responses to pain and stress, such as adrenaline (see Chapman et al., 2008). Indeed, the initial hour of the tattoo session is believed to be less variable, both sensorially and emotionally. A1 stated that "adrenaline suppresses pain for the first couple of hours. After it wear offs, they feel more pain. They also feel a chemical change in their brain where their emotion can change. For example, they can feel depressed or hollow" (A1).

Considering the excerpts and field observations, a 1-h window for examining longitudinal changes in pain ratings during VR use was deemed appropriate and logistically plausible. Ultimately, this insight narrowed the selection criteria of VR simulations by providing a valid time constraint, which we elaborate upon in the methods section of the field experiment.

\section{Tattoo and the Pain Experience}

Qualitative interviews were also used to identify the factors responsible for variability in pain associated with tattoo procedures. Aside from individual differences in pain tolerance, the pain experienced by the individual was determined by three major factors: location of the tattoo, the type of tattoo machine, and the style of the artist.

\section{Pain Sensitivity Taxonomy}

Tattoo artists and customers with pre-existing tattoos were asked to identify which areas of the body were most sensitive to pain during a tattoo. Responses indicated clear distinctions of pain sensitivity across various parts of the body. For example, A2 stated that "anywhere where there isn't a lot of muscle or fat will hurt more than other spots, like your sternum or elbow. The wrist is also a tender location for a tattoo." A3 articulated that "Face tattoos hurt like hell. Obviously, any tattoo in private areas will hurt the most." C2 mentioned that "shoulders and biceps don't hurt as much but certain spots hit a nerve at times."

Qualitative data suggests that there are three broad levels of pain sensitivity as it relates to tattoo pain. Because surveyed artists had tattoos across all regions depicted in Figure 1, they provided valid first-hand accounts of the pain associated with each region. Consensus among interviewed artists as to the sensitivity ratings depicted in Figure 1 lend further support to this taxonomy of tattoo pain. In sum, the location of a tattoo significantly dictates the level of pain one experiences during the procedure, and there are generally three levels of severity based on that location (high, mid, and low). These designated areas are in line with previous work on heat-related pain thresholds across body regions (see Park et al., 2019).

\section{Types of Tattoo Machines}

A tattoo machine (or "tattoo gun") is considered a surgical tool comprised of an oscillating needle which penetrates the skin to embed colored ink, albeit at two different settings: outlining and shading. Based on observational and interview data, outlining is used to create contrast in a tattoo, and involves deeper penetration into the skin and darker colors. Conversely, shading involves broader strokes to fill in empty space on the skin with color. Two separate guns are typically used for either style; each with a different pain threshold associated with it. Consensus among the artists interviewed was that lining is more painful than shading. A1 articulated this point by stating that "there are 


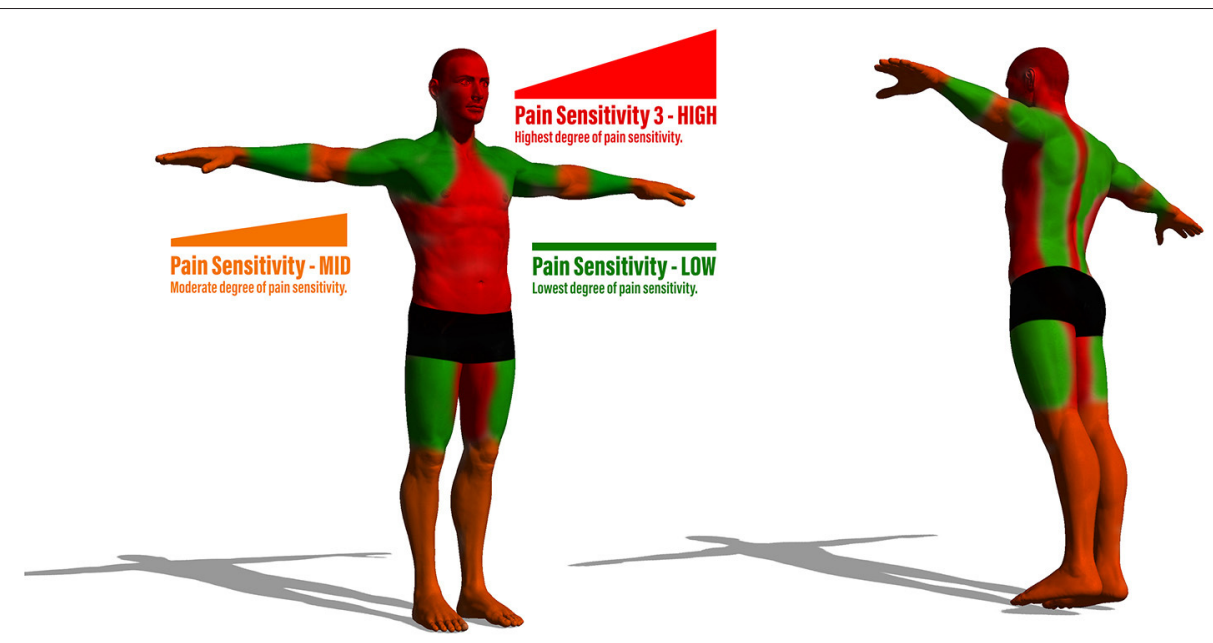

FIGURE 1 | Visualization of the two experimental conditions wherein the VH either responded or ignored an event occurrence during the interaction.

several, mainly two kinds of processes during a tattoo: lining and shading. Some say lining is more painful than shading, but it can vary."

Shadowing further provided insight into the timeline for tattoos, especially for larger ones, such that artists generally spend the first portion of a session on outlining. In fact, larger tattoos often demand the entire first session to be dedicated to the outline, having the customer return weeks later for shading after the outline has healed. In this way, researchers have some control over pain variability due to the first hour of most tattoos being comprised of outlining rather than a variant combination of the two across potential participants. In sum, this artifact the procedural consistency of artists outlining before shading allows for researchers to control for type of gun used during data collection.

\section{Artist Style}

Several artists and customers recalled multiple tattoo procedures wherein a high degree of pain came because of their "heavyhandedness." Several artists had reputations for being gentle with customers, while others were known for pressing the gun down on the skin with greater force, thereby inflicting greater pain on the customer. This observation informed the subsequent experimental design such that only one artist was used. A1 stated that "tattoo artists can have heavy hands, which can influence the pain. Some are gentle, and others are rough. The machine can also influence pain, but not to the degree as the artist."

\section{Media Use During Tattoos}

Media use is part and parcel of the modern tattooing experience. In addition to artists stating that music is typically playing in the background of their workspaces, artists also mentioned that customers regularly use smartphones during their sessions. This was further supported by observational data which showed that all customers, at various points throughout the tattoo, used their smartphones. Common uses included popular social media applications and texting. However, music was the most common form of media consumed during a tattoo. Music selection is typically controlled by the artists, though they regularly offered users the ability to connect their phones or suggest songs. To this point A1 states, "About half of my customers use headphones during their tattoo. The second most common activity is them on their phones, using social media." Use of more interactive media was also alluded to by C2 who stated, "I would love to play (video) games during my tattoo, but that would probably distract me too much" (C2).

\section{VR User and Tattoo Artist Considerations Reactivity}

Reactivity deals with issues related to movement during a VR experience. Artists expressed concern for ensuring that movement is limited during the procedure, as any sporadic twitches or body shifts can influence the quality of the tattoo. That is, any interactivity wherein the user would engage in swift or sudden movements would become problematic for the artist. This could be particularly problematic when considering that tattoos around the shoulder are sensitive to sudden head movements, which are common during VR experiences. As a result, any immersive experience inciting sudden head movements would not be ideal.

\section{Body Position}

Developers seldom account for user body positioning while using VR. While the Netflix application for the Oculus Go headset contains a "car mode" where content position adapts to users' head orientation, seldom is this principle applied to VR games. Indeed, all VR experiences tested in the context of pain research require the user to be somewhat upright and forward-facing. Positioning also refers to the varied positions that individuals must assume during a tattoo, all of which assume different points along the $\mathrm{x}$ - and $\mathrm{y}$-axis. As demonstrated in Figure 2, tattoos in the same general area (shoulder) may require the recipient to change their positioning, preventing use of a conventional, upright VR posture. 


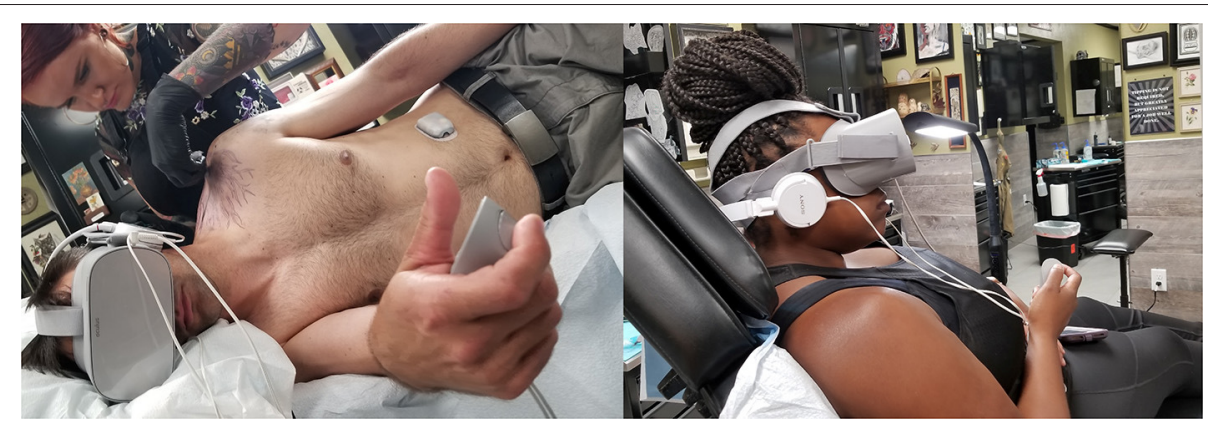

FIGURE 2 | Graphic representation of the experimental procedures.

\section{Duration}

One point of concern mentioned by one tattoo artist addressed the duration of the VR experience used. As previously mentioned, the timeline of a tattoo is typically structured in hour-long sessions, with breaks in-between. One artist inquired as to whether one standalone VR experience could entertain customers, or whether there would be multiple experiences to comply with that time frame. The average smartphone-based VR session in 2018 lasted $28.75 \mathrm{~min}$, whereas PC-based VR HMDs (e.g., HTC Vive, Oculus Rift) yielded an average session time of $45.91 \mathrm{~min}$ (Technalysis Research, 2018). While session data may comprise use of multiple simulations, the data suggests that VR experiences can provide sufficient content for an ecologically valid length of time (i.e., $45 \mathrm{~min}$ to an hour).

\section{Existing Tattoos}

Important to mention is apprehension felt by artists to take on new customers should they be using VR. That is, because individuals can faint because of the pain from a tattoo, artists typically do not know how one will react. Because of this, one artist suggested that any experiment involving customers would be best served by focusing on people with preexisting tattoos, assuring the artist that the customer is familiar with the level of pain associated with the procedure. Another artist shared a similar sentiment, stating that she would feel comfortable only using customers with pre-existing tattoos. The primary rationale being that new customers may not know how they will respond physically to the tattoo experience. As A1 articulated, "This is the best way to go because with new customers, or people with less than like five tattoos, a headset will prevent me from catching signs of passing out."

\section{Mental Transportation}

Artists and customers were asked to describe in detail the various ways they cope with pain during the tattoo experience. While many mentioned that media-based distraction (i.e., use of smartphones) helped them cope with pain, a common theme present in their responses was the mental transportation to their "happy place" With regards to this transportation A1 stated, "I know I try to find my happy place. I'll imagine myself in a forest or something." C2 also stated, "I definitely try to imagine myself somewhere else. We all try to find our little "happy place."

\section{VR Simulation Selection Criteria}

The qualitative data helped established a valid inclusion criteria for (a) the selected VR intervention used in the subsequent field experiment and (b) the participants to undergo the intervention. First, a VR simulation must accommodate a wide variation in its users' body positions, some of which may have the user lying face down, or on their side. Because many simulations require the user to be forward-facing and standing erect, using these simulations in these positions may be disorienting and cause sickness and discomfort. One potential solution is to present a virtual environment void of strong gravitational forces, thus allowing users to experience the environment naturally regardless of their head orientation and placement along the $\mathrm{x}$ - and $\mathrm{y}$-axes. The only two naturally occurring environments where this is the case is underwater and in space (zero gravity). Among these two options, an underwater experience presents the most viable choice because it (a) provides more objects with which the user can interact with, and (b) presents a more comfortable and familiar setting matching the aforementioned "happy place" descriptions.

As it relates to the participant selection, the location of the tattoo and its relative pain sensitivity rating served as primary exclusion criteria. High pain sensitivity areas may include private areas of the body, which would introduce privacy concerns. Furthermore, tattoos on the head and neck region would prove problematic considering the use of an HMD. Areas of moderate sensitivity are also challenging because they may include points that may trigger reflexive responses that may disorient the user (e.g., wrist, elbow). Low pain sensitivity areas thus presented the best option because (a) they are areas most commonly tattooed, and (b) are not in regions that would typically interfere with VR use (e.g., hand tattoos would prevent use of a VR hand controller). Table 1 synthesizes this information and serves as a guide for determining what VR content is most conducive to a particular tattoo procedure based on pain and mobility considerations.

In sum, the findings informed the selection of a simulation that was (a) interactive yet limited in physical exertion/reactivity (e.g., head movement), (b) agnostic of the user's body position and orientation, and (c) provided the user with sufficient content to experience for the duration of the procedure. The only commercially available VR simulation that met this set of criteria, 
TABLE 1 | Design considerations for designing VR content for use during a tattoo procedure.

\begin{tabular}{llll}
\hline Tattoo location & Pain sensitivity & Can use VR? & Limitations to simulation \\
\hline Head & High & No & N/A \\
Neck & High & No & N/A \\
Traps & High & Maybe & D, F \\
Chest & High & Maybe & F, U \\
Shoulders & Low & Yes & F, 1C \\
Biceps/Triceps & Low & Yes & F, 1C \\
Elbow & Mid & Yes & D, F, 1C \\
Forearm & Low & Yes & F, 1C \\
Hand & Mid & Yes & F, 1C \\
Armpits & High & Maybe & F, OC \\
Ribs & High & Maybe & F, U, OC \\
Stomach & High & Maybe & F, U, OC \\
Groin & High & No & N/A \\
Outer-thigh & Low & Yes & None \\
Inner-thigh & High & Maybe & None \\
Knee/Shin & Mid & Maybe & None \\
Calf & Mid & Maybe & D \\
Feet & Mid & Yes & None \\
\hline F, Forward-facing; & Ho & D, &
\end{tabular}

F, Forward-facing; U, Upward-facing; D, Downward-facing; 1C, limited to 1 Hand Controller; OC, no controller should be used. Bold values indicate the tattoo location is eligible for VR use.

among those surveyed, was "Ocean Rift," a scuba diving simulator designed for use with the Oculus Go HMD. In the following section we present a field experiment comparing the analgesic effects of experiencing "Ocean Rift" during a tattoo procedure with a control group. We provide a detailed description of the experimental procedures and measures, informed by the qualitative findings, followed by an overview of results, concluding with a discussion of the implications.

\section{EXPERIMENTAL METHODOLOGY}

The following section details a field experiment conducted to test (a) how VR influences volitional pain experienced during a tattoo, (b) whether analgesic effects facilitated by VR experiences are consistent throughout an immersive experience, and (c) the general feasibility of VR use within the context of body modification procedures (i.e., tattooing).

\section{Participants}

Based on the results from the qualitative investigation we created the following selection criteria for participants. First, participants had to have preexisting tattoos. Second, the tattoo being administered during the intervention had to be on a region of the body with the lowest pain sensitivity rating as designated in Figure 1. Third, participants had to be receiving a tattoo which would not require them to lay on their stomach. This is because existing VR simulations seldom require the user to face down. Should a simulation be given to a participant with a downwardfacing position, this would cause unnecessary neck strain. Lastly, eligible customers had to be receiving a tattoo that would take $\sim 1$ h or more to complete. This would allow the researchers to collect adequate longitudinal data during the session (i.e., pain ratings throughout the procedure).

In total, $16\left(\mathrm{M}_{\mathrm{age}}=28.38, \mathrm{SD}=8.5\right)$ customers met the selection criteria and participated in the field experiment. Of the 16,9 were randomly assigned to the control condition. Half of the participants identified as male, whereas the other half identified as female. Each participant received a tattoo on a part of their body deemed low on pain sensitivity as outlined in the qualitative study, with nine (9) receiving tattoos on their non-dominant arm/shoulder, four (4) on their dominant arm/shoulder, two (2) on their left leg/thigh, and one on the upper back.

\section{Recruitment Procedures}

As previously mentioned, tattoo artists vary in terms of how much pressure they apply to the skin during procedures, a factor known as "heavy-handedness." To control for differences across heavy-handedness, we selected one specific artist to tattoo the eligible participant pool. The artist was regionally and nationally renowned, with over a decade of tattooing experience across all regions of the body. Participants would all be customers who sought tattoos at Body Tech, a tattoo parlor in North Central Florida, and were either (a) assigned to the artist by the shop, or (b) sought out the artist specifically for the tattoo. The artist was given IRB-approved flyers to hand out to potential customers meeting the selection criteria during the summer of 2018 (June through August). Because customers must meet with the tattoo artist for a consultation prior to the date of the actual tattoo, this period was used to inform the eligible customer of the opportunity to participate in the study. If the customer optedin, the artist would notify the researcher of the date/time of the appointment, allowing the researcher to meet the participant prior to the tattoo and inform them further of the study.

\section{Stimulus}

The VR simulation used as the experimental manipulation met all of the aforementioned criteria: limited reactivity, agnostic of body position, nature-related, and containing at least an hour of content. Based on a thorough review of paid and free VR simulations on the Oculus store, the only simulation which met the criteria was "Ocean Rift." Ocean Rift is a VR simulation advertised as an aquatic safari park. The simulation allows the user to explore underwater biomes of various species, such as Dolphins and Seals. Users may select a specific biome and explore a large underwater territory as a scuba diver using the Oculus Go hand controllers. Because the simulation is underwater, using the HMD while laying one's side should not disorient or confuse the user. This is because underwater submergence elicits a sense of weightlessness similar to zero-gravity, hence why underwater tanks are often used to provide astronauts with zero gravity training. Given the nature of the simulation, users may explore the environment regardless of head orientation shifting along the $\mathrm{x}$ - or y-axis (Figure 2).

\section{Independent Measures}

The absence or use of VR during the tattoo procedure constituted the experimental manipulation. Participants in the control group 
were not explicitly instructed to use or consume a specific piece of media content on a particular platform during the procedure, as they were to be examined in their natural state during a tattoo. However, all participants were ultimately exposed to similar types of media content, both through use of their smartphone and via the music played by the artist. In the VR condition, participants played the "Ocean Rift" VR simulation during the procedure.

\section{Control Measure: Pain Anxiety}

Anxiety prior to beginning the tattoo procedure was measured by adapting Marteau and Bekker's (1992) State-Trait Anxiety Inventory (STAI) scale. The six-item short-form scale assesses participants' level of agreement with various statements reflecting their feelings prior to the tattoo, including "I feel calm," "I feel relaxed," and "I feel upset" (reverse coded).

\section{Dependent Measures}

\section{Pain Resilience}

Pain resilience served as a within-subjects repeated measure, assessed pre- and post-intervention. Slepian et al. (2016) 5-point, 14-item scale (Slepian et al., 2016) was used, asking participants to rate how often they react to prolonged pain in various ways, with (0) representing "Not at all" and (4) representing "All the time." Items consisted of "I push through it" and "I try to stay relaxed," among others.

\section{Media Enjoyment}

Media enjoyment was measured using a 5-item, 7-point Likert scale adapted from commonly used items in HCI research (Oliver et al., 2007; Guillory and Sundar, 2014; Hurst et al., 2016). Participants rated their level of agreement with various statements about the media they were exposed to during the tattoo, with (1) representing "Strongly disagree" and (7) representing "Strongly agree." Statements included "I enjoyed the media I consumed," "The media I used was exciting," and "The media I used was boring," among others. Participants in the VR condition were instructed to rate their enjoyment of the simulation itself.

\section{Self-Reported Pain}

Tattoo pain ratings were assessed using a 10-point numerical rating scale (NRS) commonly used in VR and pain research (Indovina et al., 2018). Participants were asked to verbally rate their level of pain with (0) representing "no pain whatsoever," and (10) being "the worst pain ever experienced." This served as a within-subjects longitudinal outcome as it was collected six (6) times throughout the tattoo experience. The scale also served as a means by which to collect the overall pain experienced during the tattoo, and pain expectancy collected prior to the procedure.

\section{Follow-Up Data}

A week after the initial session, the researcher contacted the customer via e-mail with a link to a follow-up survey measuring the degree to which they felt media distracted them from pain, pain resilience, and an overall pain rating for the 1-h session. Furthermore, as a measure of customer satisfaction, participants were asked to rate the likelihood of getting another tattoo in the future, whether they would they would get that tattoo at Body Tech.

\section{Procedures}

Eligible customers meeting the inclusion criteria of the study would be noted by the artist, with their information given to the researcher upon confirmation of an appointment. Once the appointment date/time was provided for the session, usually several days after the initial confirmation, the researcher arrived $15 \mathrm{~min}$ prior to the session. Per the tattoo shop's operating procedures, customers used this 15-min window to meet with the tattoo artist, review the design, and confirm its placement on the body. Upon authorization from the customer, the artist then prepared their workstation for the tattoo. During this preparation, the customer was introduced by the artist to the researcher. Afterwards, both the researcher and the customer relocated to a private waiting area as the artist prepared the station. At this time, the researcher explained details about the study, including general details about the general procedures along with an informed consent form. If the customer agreed to participate, the researcher then provided them with a pre-questionnaire assessing demographic information, pain resilience and pain expectancy, and other measures.

It should be noted that the researcher randomly assigned each participant to either the control or VR condition prior to the initial encounter. It was only after completing the prequestionnaire that the participant was informed that they would either (a) be observed in their natural setting during their tattoo, or (b) be asked to try a VR simulation during the procedure. Participants in the control condition were told the researcher would merely be sitting alongside them, observing their tattoo experience, and periodically asking them to rate their level of pain during the tattoo.

Across both conditions, pain ratings were only collected after the first $15 \mathrm{~min}$ of the tattoo because this window served as a monitoring period for the artist. After the first $15 \mathrm{~min}$, the researcher then verbally inquired as to the participant's pain rating every $10 \mathrm{~min}$. The time increments between ratings varied slightly across participants due to the fact that (a) pain ratings had to be collected when the tattoo gun was not actively penetrating the skin, and (b) the intervals of time the artist would keep the gun on the skin would vary between short bursts of 2-4 s and longer outlining gestures which could last between 15 and 30 s. Furthermore, observations revealed that, during outlining or shading, artists may only lift the tattoo gun for a short period of time before commencing penetration of the skin. The ideal instance for the researcher to ask for a participant's pain rating is when the gun is lifted off of the customer's skin for over $10 \mathrm{~s}$. This usually occurs when the artist takes the gun to their paint station, located directly behind them, to reload the ink onto the needle. This event takes roughly between 10 and $15 \mathrm{~s}$ and occurs every couple of minutes during the tattoo as the ink needs to be regularly replenished. As a result, the researcher would only ask for pain ratings during this window of time, leading to slight variations in time between pain ratings. The researcher would remain with the customer during the initial uninterrupted session 


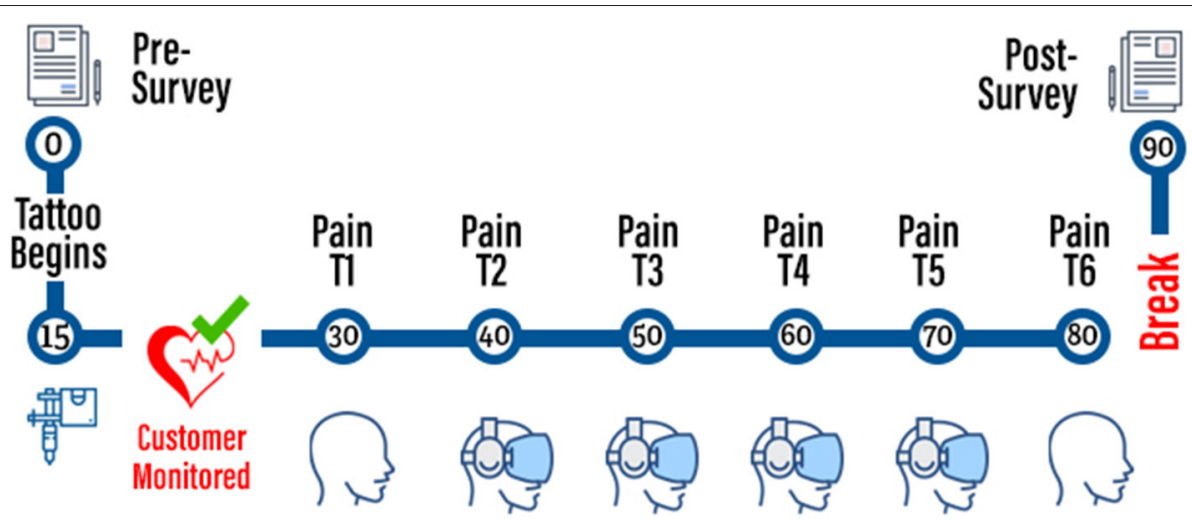

FIGURE 3 | Six randomly generated VHs pretested prior to the stimuli development.

(between 60 and $90 \mathrm{~min}$ ). As shown in the qualitative data, the timeline for a typical tattoo is broken into 1-h increments, with 15-min breaks interspersed. Thus, pain ratings were collected at six different times during the first hour.

If the participant's initial hour session exceeded the time needed for six ratings to be yielded, the researcher remained seated, taking notes related to the customer's experience, until the artist initialized a break. Once the artist initialized a break, the customer's tattoo was bandaged, and they were then instructed to complete a short paper-and-pencil questionnaire measuring dependent variables of interest. After completing the questionnaire, which took roughly $2 \mathrm{~min}$ to complete, the researcher thanked the customer for their participation and left the tattoo shop.

In the VR condition, the procedures were exactly the same as in the control condition, with two major differences. First, after participants signed the consent form and were informed of their use of VR, the researcher allowed the user to test the simulation for several minutes. During this demo the participant familiarized themselves with proper use of the HMD and hand controller. Once the user felt comfortable using the equipment, the researcher retrieved the HMD and controller, and both waited for the artist to complete the set-up and initiate the tattoo. The second major difference related to the use of VR during the tattoo procedure. The first pain rating was collected at roughly minute 30 without use of VR, as in the control condition. This was done to establish a baseline pain rating across both groups. After the initial pain rating, the researcher would then notify the artist that they would equip the participant with the HMD, hand controller, and headphones. Once the customer verbally confirmed that they had begun the simulation, the tattoo procedure would continue. Pain ratings were then collected once every $10 \mathrm{~min}$ while the customer used "Ocean Rift," leading to a total of 4 pain ratings while in VR. After the 5th pain rating, roughly at minute 70 , the researcher informed the participant that they would remove the VR equipment, and that the tattoo would continue. Afterwards, a final (6th) pain rating was collected at roughly minute 80 , upon which a break would ensue. Procedures for the VR condition are depicted in Figure 3.

\section{Experimental Results}

\section{Pain Anxiety}

Participants in the control condition $(M=6.01, \mathrm{SD}=0.67)$ did not significantly differ in their anxiety prior to the tattoo procedure (cronbach's $\alpha=0.82$ ) from those in the VR condition $(M=5.92, \mathrm{SD}=1.43, \mathrm{SD}=1.43), t_{(14)}=0.24, p>0.05$.

\section{Pain Resilience}

Independent sample $t$-tests revealed no statistically significant differences between the control and VR group in terms of pain resilience (cronbach's $\alpha=0.96$ ) prior to the tattoo session (T1) $t_{(14)}=-0.447, p>0.05$. Similarly, there were no differences in resilience between groups at after the session (T2) $t_{(14)}=$ $-0.91, p>0.05$. However, paired samples $t$-tests found that VR significantly increased pain resilience from $\mathrm{T} 1$ to $\mathrm{T} 2 t_{(6)}=-2.05$, $p<0.05$, whereas resilience remained unchanged among those in the control group $t_{(6)}=-0.16, p>0.05$.

\section{Media Enjoyment}

Independent sample $t$-tests revealed no statistically significant differences in media enjoyment (cronbach's $\alpha=0.67$ ) between the control $(M=4.5, S D=1.23)$ and VR group $(M=4.97, \mathrm{SD}=$ $0.43), t_{(14)}=-0.89 p>0.05$.

\section{Self-Reported Pain}

\section{Anticipated Pain}

There were no significant differences in the level of anticipated pain between the control $(M=5.11, S D=1.19)$ and VR condition $(M=6.14, S D=1.57) t_{(14)}=-1.15, p>0.05$.

\section{Pain During a Tattoo}

Participants in the control condition reported significantly less pain at $\mathrm{T} 1(\mathrm{M}=3.78, \mathrm{SD}=2.22)$ than participants in the VR condition $(\mathrm{M}=5.71, \mathrm{SD}=0.76), t_{(14)}=-2.19, p<05$. We discuss potential reasons for differences in baseline pain ratings in the discussion section. To assess the analgesic effects of VR, independent samples $t$-tests were conducted to determine whether changes in pain from $\mathrm{T} 1$ to $\mathrm{T} 2$ were substantially different between the groups. Use of VR significantly reduced self-reported pain from $\mathrm{T} 1$ to $\mathrm{T} 2\left(M_{\text {Difference }}=1.14, S D=2.19\right)$, 
TABLE 2 | Raw pain ratings collected throughout the tattoo process across groups.

\begin{tabular}{lccccccr}
\hline Group & T1 & T2 & T3 & T4 & T5 & T6 $^{\text {T3 }}$ & T7 $^{\text {* }}$ \\
\hline Control & $3.7^{\mathrm{a}}$ & 4.4 & 3.3 & 3.3 & 2.8 & $3.3^{\mathrm{a}}$ & $4.2^{\mathrm{a}}$ \\
VR & $5.7^{\mathrm{b}}$ & $\mathbf{4 . 5}$ & $\mathbf{4 . 1}$ & $\mathbf{4}$ & $\mathbf{4}$ & $5.2^{\mathrm{b}}$ & 4.2 \\
\hline
\end{tabular}

Different superscript letters indicate statistical significance.

${ }^{\star} T 7$ represented the overall pain rating given by each participant at the conclusion of the 1-h session.

${ }^{\star}$ Mean scores from eight participants who completed follow-up survey, evenly distributed across groups. Bold values indicate ratings were measured while the participant was using VR.

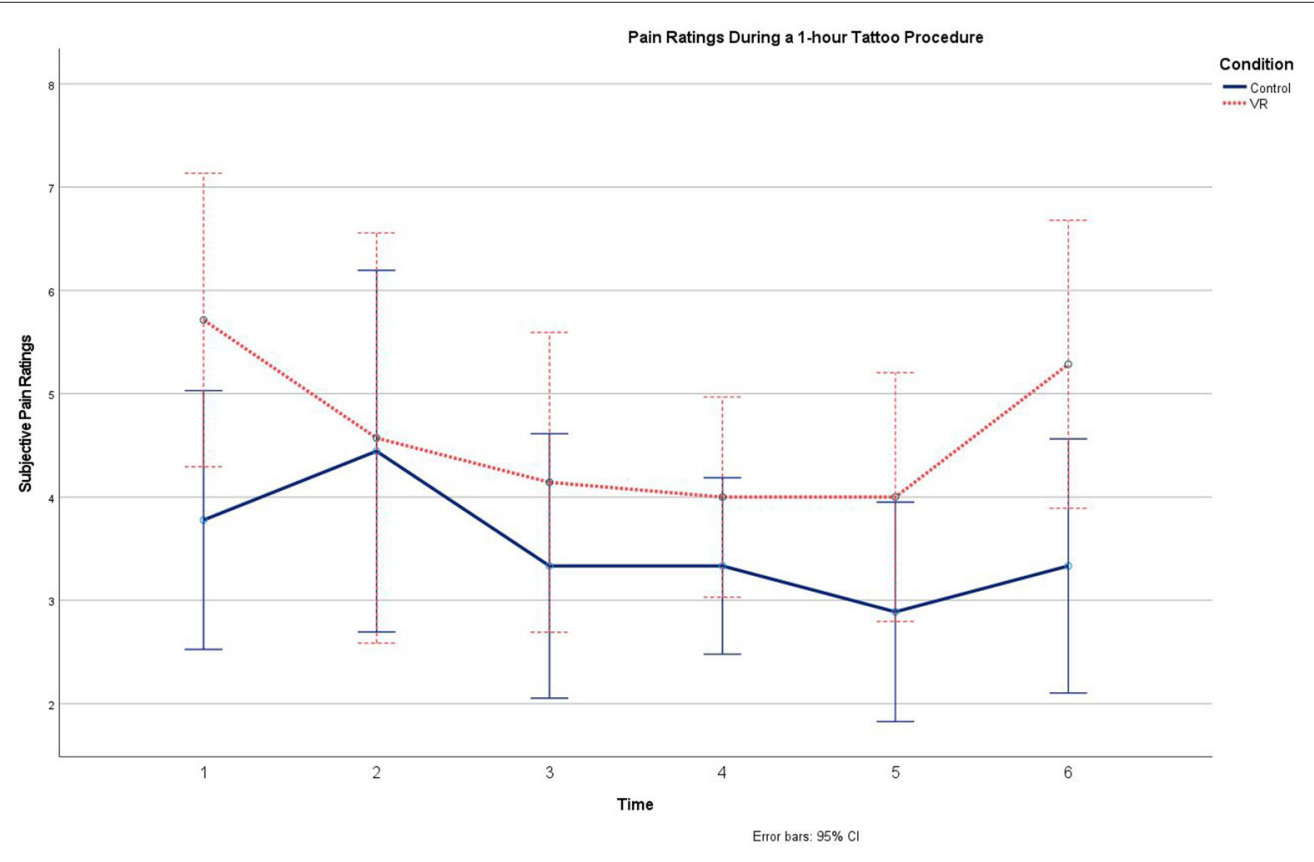

FIGURE 4 | Graph demonstrating the interaction effect between time and contextual responsiveness on copresence with a VH.

whereas pain ratings increased during the same time frame for those in the control group $\left(M_{\text {Difference }}=-.66, S D=1.8\right) \cdot t_{(14)}=$ $-1.81, p<0.05)$. From T2 through T5, a series of independent samples $t$-tests revealed no significant differences in self-reported pain between groups (all $p$ 's $>0.05$ ). However, it should be noted that at T6, when participants in the VR condition had removed their HMD, there was a significant difference in pain rating between those in the VR condition $(\mathrm{M}=5.29, \mathrm{SD}=1.8)$ and those in the control condition $(\mathrm{M}=3.33, \mathrm{SD}=1.6), t_{(14)}=$ $-2.25, p<0.05$.

To evaluate VR's longitudinal impact on perceived pain during a tattoo, a repeated measures analysis of variance (ANOVA) was conducted. The analysis compared changes in pain ratings throughout the tattoo procedure across groups (Table 2). In the control group, within-subjects contrasts revealed no significant differences between the 6 pain ratings collected during the hourlong session, exhibiting neither a linear $\left[F_{(1,8)}=1.35, p>0.05\right.$, $\left.\eta^{2}=0.11\right]$ nor quadratic trend $F_{(1,8)}=0.202, p>0.05, \eta^{2}$ $=0.02$ ). However, pain ratings for the VR group exhibited a significant quadratic trend $\left.F_{(1,6)}=7.69, p<0.05, \eta^{2}=0.56\right)$. A quadratic distribution (Figure 4) suggests that pain (a) declined when VR was introduced, (b) was stable during immersion, and (c) spiked upon removal of the HMD.

\section{Overall Pain Recall}

An independent Samples $t$-test revealed that participants in the control condition rated their overall pain during the session significantly lower $(M=4.22, S D=1.72)$ than those who used VR $(M=5.86, S D=1.22) t_{(14)}=-2.13, p<0.05$. However, both groups' overall pain ratings $(M=4.94, S D=1.69)$ were significantly higher than their average scores provided during the session $(M=4, S D=1.39), t_{(15)}=3.83, p<0.01$. This is further discussed in the discussion section.

To test the assumption that pain resilience may influence the direct effect of VR on participants' overall pain rating, a bootstrapped (5,000 resamples) moderation analysis (Hayes, 2015) was conducted via the PROCESS macro (Model 1) (Hayes, 2012). The moderation model revealed a non-significant interaction between pain resilience and the intervention, dummy coded as 0 for control and 1 for VR. The $\mathrm{R}^{2}$ change in the 
regression equation when change in pain resilience from $\mathrm{T} 1$ to T2 was included as a moderator $(\Delta F=3.65, p=0.08)$ indicates that the conditional effects of the VR intervention on overall pain ratings were not based on changes in pain resilience. When pain resilience decreased, VR did not significantly influence overall pain ratings $(b=-0.38, S E=1.35, p>.05$; 95\%CI: $-3.33,2.56)$. However, when pain resilience increased, VR led to significantly lower overall pain ratings compared to the control condition ( $b$ $=-4.78, S E=1.11, p=0.01$; 95\%CI: 0.86, 5.72).

\section{Follow-Up Pain Recall}

To test the assumption that pain resilience may influence the direct effect of VR on pain recall a week after the intervention, another bootstrapped (5,000 resamples) moderation analysis was conducted. The moderation model revealed a significant interaction between pain resilience and the intervention. The significant $\mathrm{R}^{2}$ change in the regression equation when change in pain resilience was included as a moderator $(\Delta F=57.95, p<$ 0.01 ) indicates that conditional effects of the VR intervention on pain recall were based on changes in pain resilience. When pain resilience decreased, VR led to greater pain recall compared to the control condition $(b=4.86, S E=0.68, p<0.01$; 95\%CI: 2.97 , 6.76). When pain resilience increased, VR led to less pain recall compared to the control condition $(b=-2.43, S E=0.52, p<$ 0.01; 95\%CI: $-3.89,-0.97)$.

\section{Follow-Up Data}

Follow-up data was collected and evaluated via emails sent to all participants a week after their scheduled appointment. Of the 16 participants contacted, only half $(N=8)$ replied and completed the follow-up questionnaire, four from each condition.

\section{Distraction}

Participants in the control condition $(M=3.5, S D=1.91)$ did not differ significantly from those in the VR condition $(M=5.5, S D$ $=1$ ) in terms of how much they felt the media (e.g., VR, music) helped distract them from tattoo pain $t_{(6)}=-1.85, p>0.05$.

\section{Resilience}

Among participants in the control group, pain resilience remained stable from post-intervention $(M=43.5, S D=8.34)$ to follow-up $(M=42.25, S D=6.34) t_{(3)}=1, p>0.05$. For participants in the VR condition, pain resilience significantly declined from post-session $(M=44.5, S D=12.23)$ to the follow-up $(M=37.5, S D=8.58) t_{(3)}=-3.3, p<0.05$.

\section{Attitudes Toward the Tattoo Shop}

Participants who did not use VR during their tattoo expressed favorable attitudes (Cronbach's $\alpha=0.99$ ) toward the tattoo shop $(M=6, S D=1.37)$ as did participants who used VR during the tattoo $(M=6.25, S D=0.57), t_{(6)}=-0.34, p>0.05$.

\section{Likelihood of Receiving Another Tattoo}

Participants expressed similar levels of intention with regards to getting another tattoo $\left(\mathrm{M}_{\text {control }}=2.5, \mathrm{SD}=3 ; \mathrm{M}_{\mathrm{VR}}=1.25, \mathrm{SD}\right.$ $=0.5), t_{(6)}=0.82, p>0.05$. However, participants who did not use VR during their tattoo less likelihood of receiving a tattoo at the tattoo shop $(\mathrm{M}=1, \mathrm{SD}=0)$ than participants who used VR during the tattoo $(\mathrm{M}=2.25, \mathrm{SD}=0.96), t_{(6)}=-2.61, p<0.05$.

\section{GENERAL DISCUSSION}

The mixed-methods investigation yielded rich insight into how HCI and pain scholars can test VR interventions on subjects experiencing tattoo pain. By identifying safety, procedural, and usability factors specific to the tattoo experience, we present guidelines for effectively testing immersive content on subjects receiving a tattoo. We then empirically tested the efficacy of this methodological paradigm by conducting a field experiment wherein pain ratings of tattoo recipients were compared between a VR intervention and control group which used non-immersive media. Overall, results present methodological, theoretical, and practical contributions benefitting VR scholars interested in testing immersive content during a common but understudied scenario.

\section{Feasibility of VR During Tattoo Use}

The procedures, and overall methodological approach, used in in this study serve as an effective guideline for future testing of VR interventions among tattoo recipients. Indeed, considering the delicacy of the tattooing procedure, considerations raised in this investigation help researchers properly build and test immersive content on tattoo recipients. More importantly, the field experiment extended the analgesic effects of VR to a type of pain yet studied by VR-based pain management researchers. Whereas previous work has shown VR can reduce pain associated with acute injuries (Kipping et al., 2012), chronic conditions (Gromala et al., 2015), and disease (Wright et al., 2005), this study extends these benefits to volitional pain felt during a tattoo procedure. By validating tattoo recipients as a valid subject pool to test VR-based pain management interventions, we open a large and ecologically valid participant pool for use in future research. Moreover, this effort addresses the call for more experiments "involving maintenance or continuation of behavior while individuals are in pain” (Slepian et al., 2016) (p. 461).

This work also establishes tattoo recipients, and by extension any individual seeking body modification, as a viable end user of VR content. To the best of our knowledge this is the first formal investigation into how humans experience VR content during painful body modification. Considering that participants responded favorably to the experience, and that this segment of the population is believed to be increasing (Sapp et al., 2019), it is evident that this demographic warrants attention, both by VR content developers and researchers interested in studying how immersive content influences real-world pain perception.

\section{Theoretical Implications: Pain Resilience}

Results from the field experiment provide further empirical support for the analgesic effects of VR, while simultaneously highlighting potential factors driving these effects. Extant research has largely explained these benefits by arguing that VR's elicitation of presence is a pain-reducing distraction (Gupta et al., 2018). However, this study sought to explore, at least preliminarily, alternative factors beyond distraction, namely pain 
resilience. Pain resilience is defined as the capacity to which an individual can cognitively and emotionally regulate themselves during prolonged pain, and is inversely related to subjective pain ratings with acute experimental pain (Ankawi et al., 2020). Our results support the potential for VR to increase pain resilience during a tattoo; VR significantly increased pain resilience from pre- to post-intervention, whereas resilience remained stable among those in the control condition. Furthermore, this change in resilience significantly moderated the effect of the intervention on participants' pain recall a week after the session. In sum, the ability of a VR simulation to reduce self-reported pain is significantly influenced by the ability for the simulation to increase pain resilience.

One potential explanation for the resilience change is that aspects of the simulation contributed to participants' ability to cope with pain over time. Considering that the simulation involved immersion into nature (i.e., underwater biomes), it is important to acknowledge exposure to nature as a potential explanation. Indeed, previous work has shown that exposure to natural environments can increase resilience during stressful events among children (Wells and Evans, 2003) and adults (van den Berg et al., 2010). However, extant literature on the effects of nature proves problematic if one accepts the distraction paradigm established by the majority of scholarship on VR-based pain management. Consider attention restoration theory (ART), a theoretical framework which argues that natural landscapes are effortlessly processed, thus freeing up attentional resources, and improving cognitive functioning. If distraction is indeed a major driver of VR's analgesic effects, and exposure to nature in VR would improve attentional capacity to the sensory experience, then virtually verdant environments should increase perceived pain during a tattoo, to the detriment of the user. Considering the competing mechanisms at-play, future work should compare the effects of verdant virtual experiences with other less natural simulations, elucidating the degree to which cognitive and affective processes are driving VR-based pain relief.

\section{Pain Management}

A vast majority of VR-based pain management studies have only focused on differences between pre- and post-intervention pain scores (Hoffman et al., 2000; Patterson et al., 2010; Schmitt et al., 2011; Gromala et al., 2015; Tashjian et al., 2017). However, this study investigated the effects of VR on pain ratings collected throughout a painful experience. As a result, we were able to collect multiple pain ratings throughout a painful experience while limiting error variance commonly found in extant literature (e.g., pain location, intensity, type). In doing so, we demonstrated that (a) VR reduced pain ratings, (b) pain remained stable throughout immersion, and (c) pain ratings spiked upon removal of the HMD. Of particular interest is how this quadratic trend in subjective pain (Figure 4) differed for those in the control group, and the implications this has on pain management. Previous literature has shown that humans cope with repetitive painful stimulation by habituating to the pain (Rennefeld et al., 2010), though this was not found in our sample. One explanation is that, while habituation can reduce subjective pain ratings, this mechanism is sensitive to the location of the pain. Indeed, studies testing the effects of consistent pain stimuli on perceived pain have prevented habituation by slightly moving the pain source (e.g., laser stimulation) (Babiloni et al., 2006). In other words, participants may have been unable to effectively habituate pain due to the tattoo gun's ever-changing point of contact.

$\mathrm{VR}$ is effective in maintaining an already reduced level of subjective pain during the course of painful stimulation not isolated to a specific point on the body. By making consistent pain stimulation more tolerable, VR should be considered as an investment for tattoo shops seeking to maximize the customer experience. Indeed, while not reported in the results, participants in the VR condition reported a greater likelihood of returning to the tattoo shop for another tattoo than those in the control condition, despite non-significant differences in enjoyment. However, this may be driven by a novelty effect, as participants had limited familiarity with VR. In general, participants in the VR condition vocalized their pleasure with the experience during and after use, mentioning the sense of "being there" as an attractive feature of the technology. Additionally, during VR use, many participants expressed the desire of exploring other environments, highlighting the potential for customized virtual environments to improve the customer experience in this context.

\section{Anticipatory Analgesia}

Previous work suggests that expectations of pain relief play a significant role in shaping self-reported pain intensity (see Bingel et al., 2011). It is reasonable to assume that similar expectations may have played a role in shaping baseline pain ratings in the field experiment considering that participants in the VR condition were aware they would be asked to rate their pain before, during, and after immersion into a virtual world. In this way, the observed differences in pain at T1 may be explained by analgesic expectations associated with the intervention. Moreover, the differences at T6 may also be explained by the "peak-end phenomenon," which shows that pain ratings are highly influenced by the highest pain experienced (peak) and the most recent pain experienced (see Stone et al., 2000). Indeed, with media-based pain interventions, such as music, there are a myriad of external factors that may influence pain perception (see Lunde et al., 2019 for a review). As Lunde et al. note, merely comparing a treatment with a control condition does not allow researchers to control for "factors embedded in the treatment context rather than in the (content) itself" (p. 991). Thus, future research should seek to explore multi-factorial experimental designs involving VR-based interventions for tattoo pain, thereby allowing for greater understanding of what content factors shape human responses to tattoo pain during VR use.

\section{Future Work}

This pilot study served as a preliminary investigation into the feasibility of VR use during a tattoo procedure. In doing so, we sought to address calls by previous researchers to examine the user experience in VR during different types of pain experiences (e.g., Ahmadpour et al., 2019). As such, the findings yielded rich, albeit limited, insight into how VR 
influences consumer perceptions of pain and user experience in the context of body modification. More importantly, however, the current work serves as a springboard for future scholars to engage in interdisciplinary research that explores the interplay between immersive media content and sociocultural factors unique to body modification. Specifically, we propose that future work should explore two specific topics: VR customization and the relationship between presence, pain, and meaning.

\section{Customization and the Pain Experience}

Extant literature emphasizes the benefits associated with both tailoring content to a user (personalization) and with allowing that user to modify content to match their specific needs/preferences (customization; see Kalyanaraman and Wojdynski, 2015). Specific to painful experiences, previous work has emphasized the importance of personalization and customization of VR analgesia (Pourmand et al., 2018). In the context of pediatric pain, Won et al. (2017) note the promise of customizing both the user's environment and virtual avatar (self-representation), and how these affordances ultimately offer users autonomy to create an experience best suited for their needs and preferences. This promise undoubtedly extends to volitional pain contexts, namely body modification. What is particularly intriguing about customized or personalized VR content during body modification is the capacity to integrate the symbolism of the tattoo into the immersive experience itself. That is, future work may seek to explore how immersive experiences that are thematically tied to the participant's tattoo, such as immersing the user receiving a jungle-related tattoo into a tropical environment, may influence key outcomes (e.g., pain, enjoyment). Additionally, future work may explore how tattoo progress in the real-world may interface with virtual events to provide users with real-time feedback on the experience while remaining anchored in the virtual world.

\section{Presence and Tattoo Meaning}

In many cases, a specific body modification holds significant social and cultural meaning to the individual. This meaning is rooted as much in the visual symbolism of the tattoo as in the process through which that symbol is permanently embedded into the individual's body. As Dann and Callaghan (2019) argue, "The "authentic" tattoo is a relational object, embedded in personal narratives and the positioning of strength and survival through pain" (p. 5). In this way, a tattoo's perceived meaning and authenticity is presumably tied to the pain produced by its manifestation. It is thus important for future work to explore the interplay between perceived pain and tattoo meaning, and whether VR's pain-reducing effects may negatively influence customers' perceptions of the tattoo. If VR use during a tattoo reduces pain perception at the cost of symbolic value, the viability of VR use in this context may prove limited. However, as noted in the previous section, infusing aspects of the tattoo experience into the immersive content via customization or personalization may offset such effects.

\section{Methodological Considerations in VR Pain Studies}

Another important factor is the method for collection selfreported pain measures. Pian ratings were verbally given by each participant. While this is common practice in pain research (source), social desirability and other factors may indeed have influenced the ratings. That is, participants may have felt the need to report lower ratings to seem more resilient to those in the environment. Participants in the VR condition may have felt less aware of the social environment and thus less susceptible to such influences. Future work may seek to address this by allowing non-verbal submission of pain ratings by subjects. For example, studies would benefit from embedding pain rating user interface to allow users to input their rating within the simulation. A similar mediated input method may be provided for those in non-immersive conditions.

\section{Limitations}

\section{Generalizability of Tattoo Pain}

Pain, while experienced and understood by every human, is a multi-dimensional construct. That is, there are many types of pain humans experience, and because of this it is difficult to generalize our results to other forms of pain. For example, consider the complex and multi-faceted nature of chronic pain. Experiences with chronic pain do not occur in a vacuum; persistent and painful conditions occur in tandem with associated biological, psychological, sociological stressors (Chi et al., 2019). These factors are simply not at-play during volitional pain episodes like tattooing and other body modification scenarios where individuals have the capacity to stop the pain source and/or are actively able to prepare and expect the pain. Nonetheless, examining VR's analgesic effects in this unique context affords researchers an opportunity to study how immersive media can influence episodic pain perception. While such efforts may not yield insights that extrapolate to more serious pain conditions, they may reveal how VR experiences facilitate psychological responses beneficial to pain management, and thus potentially inform design of VR content in clinical situations.

\section{Thematic Interpretations}

As with any qualitative endeavor, this study's interpretation of the subjects' lived experiences related to the tattoo experience is influenced by the researcher's own experience with the subject matter. It should be noted that one of the researchers involved in this study had several pre-existing tattoos, all of which were completed at the tattoo parlor in which the study took place. While this does not represent a conflict of interest, it is important to acknowledge that this may have influenced the thematic analysis of the interview data collected from both tattoo artists and customers.

\section{Sample Size}

Another important limitation is the sample size. Meta-analyses summarizing studies on VR and pain reported sample sizes ranging from as low as 2 (Lauwens et al., 2020) to as high as 97 (Chow et al., 2020). The low sample size may be attributed to the fact that data collection occurred during the summer 
months in a city with a highly transient student population. Our sample and experimental design present the high probability of the study being underpowered, and thus interpretation of results pertaining to VR's impact on tattoo-related pain perception should be done cautiously. Moreover, while previous work has explored the role of biological sex on pain perception (e.g., Berkley, 1997), we were unable to adequately assess differences across male and female participants due to the small sample. Additionally, we were unable to assess the influence of body composition among our participants (e.g., height, weight), which may ultimately influence pain perception. Yet, as a feasibility study, this work helps establish guidelines and experimental protocols for future experiments in this context.

\section{Differences in Media Usage}

All participants in the VR condition engaged in similar levels of interactivity. However, participants in the control condition varied slightly in their use of media. While all participants in the control condition listened to similar music (controlled by the tattoo artist), cell phone use varied in terms of both duration and content. That is, participants in the control condition either used their cell phone to check social media, play mobile games, or transitioned between both. Considering that both content mobile phone use and task-switching influence distraction by imposing cognitive load (see Chen and Yan, 2016), it is unclear how variation in media use within the control condition influenced the results. However, given the wealth of research showing how varying levels of interactivity influence psychological responses to media messages (e.g., Liu and Shrum, 2009) and pain (see Wender et al., 2009), future work should explore control conditions of varying types of interactive media.

\section{CONCLUSION}

This study sought to establish a new methodological paradigm using tattoo recipients as a participant pool to test VR-based pain management interventions. Second, we sought to empirically validate this approach via a field experiment. Overall, the investigation accomplished these goals, extending the analgesic effects of VR to volitional pain, while simultaneously providing future researchers with a template for testing alternative factors beyond distraction in their interventions. From an applied perspective, this work also demonstrates that VR use is a feasible activity during body modification, extending the number of contexts wherein immersive experiences may become normalized. It is our hope that this investigation will lead to more experiments leveraging this participant pool and context. In doing so, future research may continue to elucidate the ways in which VR can help individuals going through a variety of pain experiences, acknowledging that the results may not be generalizable to clinical settings.

\section{DATA AVAILABILITY STATEMENT}

The raw data supporting the conclusions of this article will be made available by the authors, without undue reservation.

\section{ETHICS STATEMENT}

The studies involving human participants were reviewed and approved by University of Florida Institutional Review Board. The patients/participants provided their written informed consent to participate in this study. Written informed consent was obtained from the individual(s) for the publication of any potentially identifiable images or data included in this article.

\section{AUTHOR CONTRIBUTIONS}

DP contributed to the experimental design, data collection, analysis, and manuscript writing. SK contributed to the experimental design and manuscript writing. $\mathrm{RF}$ and $\mathrm{SH}$ contributed to the experimental design. All authors contributed to the article and approved the submitted version.

\section{REFERENCES}

Ahmadpour, N., Randall, H., Choksi, H., Gao, A., Vaughan, C., and Poronnik, P. (2019). Virtual reality interventions for acute and chronic pain management. Int. J. Biochem. Cell Biol. 114:105568. doi: 10.1016/j.biocel.2019. 105568

Ankawi, B., Slepian, P. M., Himawan, L., and France, C. R. (2020). The effect of pain resilience on experimental pain experience across different stimuli. Psychosom. Med. 82, 593-599. doi: 10.1097/PSY.0000000000000823

Babiloni, C., Brancucci, A., Percio, C., Del, C.apotosto, P., Arendt-Nielsen, L., Chen, A. C. N., et al. (2006). Anticipatory electroencephalography alpha rhythm predicts subjective perception of pain intensity. J. Pain 7, 709-717. doi: $10.1016 /$ j.jpain.2006.03.005

Beasley, M. (2011). Who owns your skin: intellectual property law and norms among tattoo artists. South. Calif. Law Rev. 85, 1238-1176.

Berkley, K. J. (1997). Sex differences in pain. Behav. Brain Sci. 20, 371-380. doi: $10.1017 /$ S0140525X97221485

Bingel, U., Wanigasekera, V., Wiech, K., Ni Mhuircheartaigh, R., Lee, M. C., Ploner, M., et al. (2011). The effect of treatment expectation on drug efficacy: imaging

the analgesic benefit of the opioid remifentanil. Sci. Transl. Med. 3:70ra14. doi: 10.1126/scitranslmed.3001244

Breuner, C., and Levine, D. (2017). Adolescent and young adult tattooing, piercing, and scarification. Pediatrics 140:e20163494. doi: 10.1542/peds.2017-1962

Chapman, C. R., Tuckett, R. P., and Song, C. W. (2008). Pain and stress in a systems perspective: reciprocal neural, endocrine, and immune interactions. J. Pain. 9, 122-145. doi: 10.1016/j.jpain.2007.09.006

Chen, Q., and Yan, Z. (2016). Does multitasking with mobile phones affect learning? A review. Comput. Hum. Behav. 54, 34-42. doi: 10.1016/j.chb.2015.07.047

Chi, B., Chau, B., and Ta, P. (2019). "Uses of virtual reality (VR) for chronic pain," in Guazzaroni, G., editor. Virtual and Augmented Reality in Mental Health Treatment. IGI Global. 221-240. doi: 10.4018/978-1-7998-1680-5.ch013

Chow, H., Hon, J., Chua, W., and Chuan, A. (2020). Effect of virtual reality therapy in reducing pain and anxiety for cancer-related medical procedures: a systematic narrative review. J. Pain Symptom Manage. 61, 384-394. doi: $10.1016 /$ j.jpainsymman.2020.08.016

Dann, C., and Callaghan, J. (2019). Meaning-making in women's tattooed bodies. Soc. Personal. Psychol. Compass. 13:e12438. doi: 10.1111/spc3.12438 
Dascal, J., Reid, M., Ishak, W. W., Spiegel, B., Recacho, J., Rosen, B., et al. (2017). Virtual reality and medical inpatients: a systematic review of randomized, controlled trials. Innov. Clin. Neurosci. 14, 14-21.

Davies, A. N. (2006). Cancer-related breakthrough pain. Br. J. Hosp. Med. 67, 414-416. doi: 10.12968/hmed.2006.67.8.21960

Fischer, A. A. (1987). Pressure algometry over normal muscles. Standard values, validity and reproducibility of pressure threshold. Pain 30, 115-126. doi: 10.1016/0304-3959(87)90089-3

Gerin, W., Pickering, T. G., Glynn, L., Christenfeld, N., Schwartz, A., Carroll, D., et al. (2000). An historical context for behavioral models of hypertension. J. Psychosom. Res. 48, 369-377. doi: 10.1016/S0022-3999(99) 00095-1

Gromala, D., Tong, X., Choo, A., Karamnejad, M., and Shaw, C. D. (2015). "The virtual meditative walk: virtual reality therapy for chronic pain management," in Proceedings of the ACM CHI'15 Conference on Human Factors in Computing Systems (Seoul). doi: 10.1145/2702123.2702344

Guillory, J. E., and Sundar, S. S. (2014). How does web site interactivity affect our perceptions of an organization? J. Public Relat. Res. 26, 44-61. doi: 10.1080/1062726X.2013.795866

Gupta, A., Scott, K., and Dukewich, M. (2018). Innovative technology using virtual reality in the treatment of pain: does it reduce pain via distraction, or is there more to it? Pain Med. 19, 151-159. doi: 10.1093/pm/ pnx109

Hayes, A. F. (2012). PROCESS: A Versatile Computational Tool for Observed Variable Mediation, Moderation, and Conditional Process Modeling [White paper]. Available online at: http://www.afhayes.com/public/process2012.pdf (accessed November 10, 2020).

Hayes, A. F. (2015). An index and test of linear moderated mediation. Multivariate Behav. Res. 50, 1-22. doi: 10.1080/00273171.2014.962683

Hoffman, H. G., Doctor, J. N., Patterson, D. R., Carrougher, G. J., and Furness, T. A. (2000). Virtual reality as an adjunctive pain control during burn wound care in adolescent patients. Pain 85, 305-309. doi: 10.1016/S0304-3959(99) 00275-4

Hoffman, H. G., Meyer, W. J., Ramirez, M., Roberts, L., Seibel, E. J., Atzori, B., et al. (2014). Feasibility of articulated arm mounted oculus rift virtual reality goggles for adjunctive pain control during occupational therapy in pediatric burn patients. Cyberpsychol. Behav. Soc. Netw. 17, 397-401. doi: $10.1089 /$ cyber.2014.0058

Hoffman, H. G., Patterson, D. R., Seibel, E., Soltani, M., Jewett-Leahy, L., and Sharar, S. R. (2008). Virtual reality pain control during burn wound debridement in the hydrotank. Clin. J. Pain 24, 299-304. doi: 10.1097/AJP.0b013e318164d2cc

Hoffman, H. G., Sharar, S. R., Coda, B., Everett, J. J., Ciol, M., Richards, T., et al. (2004). Manipulating presence influences the magnitude of virtual reality analgesia. Pain 111, 162-168. doi: 10.1016/j.pain.2004. 06.013

Hurst, W., de Boer, B., Florijn, W., and Tan, X. J. (2016). "Creating new museum experiences for virtual reality," in 2016 IEEE International Conference on Multimedia and Expo Workshops (ICMEW) (Ave Seattle, WA), 1-6. doi: 10.1109/ICMEW.2016.7574692

Indovina, P., Barone, D., Gallo, L., Chirico, A., De Pietro, G., and Antonio, G. (2018). Virtual reality as a distraction intervention to relieve pain and distress during medical procedures. Clin. J. Pain 34, 858-877. doi: 10.1097/AJP.0000000000000599

Kalyanaraman, S., and Wojdynski, B. W. (2015). "Affording control: how customization, interactivity, and navigability affect psychological responses to technology," in The Handbook of the Psychology of Communication Technology, ed S. Shyam Sundar (Oxford: John Wiley \& Sons, Inc.), 425-444. doi: 10.1002/9781118426456.ch19

Kenny, V. (2009). "There's nothing like the real thing": revisiting the need for a third-order cybernetics. Constructivist Found. 4, 100-111.

Kipping, B., Rodger, S., Miller, K., and Kimble, R. M. (2012). Virtual reality for acute pain reduction in adolescents undergoing burn wound care: a prospective randomized controlled trial. Burns 38, 650-657. doi: 10.1016/j.burns.2011.11.010

Kolodny, A., and Frieden, T. R. (2017). Ten steps the federal government should take now to reverse the opioid addiction epidemic. JAMA 318:1537. doi: 10.1001/jama.2017.14567
Lauwens, Y., Rafaatpoor, F., Corbeel, K., Broekmans, S., Toelen, J., and Allegaert, K. (2020). Immersive virtual reality as analgesia during dressing changes of hospitalized children and adolescents with burns: a systematic review with meta-analysis. Children 7:194. doi: 10.3390/children7110194

Law, E. F., Dahlquist, L. M., Sil, S., Weiss, K. E., Herbert, L. J., Wohlheiter, K., et al. (2010). Videogame distraction using virtual reality technology for children experiencing cold pressor pain: the role of cognitive processing. J. Pediatr. Psychol. 36, 84-94. doi: 10.1093/jpepsy/jsq063

Li, A., Montaño, Z., Chen, V. J., and Gold, J. I. (2011). Virtual reality and pain management: current trends and future directions. Pain Manag. 1, 147-157. doi: $10.2217 /$ pmt.10.15

Liu, Y., and Shrum, L. J. (2009). A dual-process model of interactivity effects. J. Advert. 38, 53-68. doi: 10.2753/JOA0091-3367380204

Lunde, S. J., Vuust, P., Garza-Villarreal, E. A., and Vase, L. (2019). Musicinduced analgesia: how does music relieve pain? Pain 160, 989-993. doi: $10.1097 /$ j.pain.0000000000001452

Lyapustina, T., and Alexander, G. C. (2015). The prescription opioid addiction and abuse epidemic: how it happened and what we can do about it. Pharm. J.

Mahrer, N. E., and Gold, J. I. (2009). The use of virtual reality for pain control: a review. Curr. Pain Headache Rep. 13, 100-109. doi: 10.1007/s11916-009-0019-8

Oliver, M. B., Kalyanaraman, S., Mahood, C., and Ramasubramanian, S. (2007). Sexual and violent imagery in movie previews: effects on viewers' perceptions and anticipated enjoyment. J. Broadcast. Electron. Media 51, 596-614. doi: 10.1080/08838150701626446

Park, S., Roh, S.-H., and Lee, J.-Y. (2019). Body regional heat pain thresholds using the method of limit and level: a comparative study. Eur. J. Appl. Physiol. 119, 771-780. doi: 10.1007/s00421-018-04068-4

Patterson, D. R., Jensen, M. P., Wiechman, S. A., and Sharar, S. R. (2010). Virtual reality hypnosis for pain associated with recovery from physical trauma. Int. J. Clin. Exp. Hypn. 58, 288-300. doi: 10.1080/00207141003760595

Phelan, I., Furness, P. J., Fehily, O., Thompson, A. R., Babiker, N. T., Lamb, M. A., et al. (2018). A mixed-methods investigation into the acceptability, usability, and perceived effectiveness of active and passive virtual reality scenarios in managing pain under experimental conditions. J. Burn Care Res. 40, 85-90. doi: 10.1093/jbcr/iry052

Pourmand, A., Davis, S., Marchak, A., Whiteside, T., and Sikka, N. (2018). Virtual reality as a clinical tool for pain management. Curr. Pain Headache Rep. 22:53. doi: 10.1007/s11916-018-0708-2

Rennefeld, C., Wiech, K., Schoell, E. D., Lorenz, J., and Bingel, U. (2010). Habituation to pain: further support for a central component. Pain 148, 503-508. doi: 10.1016/j.pain.2009.12.014

Sapp, J. L. C., Vogel, R. L., Telfair, J., and Reagan, J. K. (2019). Evaluating web-based platforms and traditional methods for recruiting tattoo artists: descriptive survey research study. JMIR Dermatol. 2:e14151. doi: 10.2196/14151

Scheffler, M., Koranyi, S., Meissner, W., Strauß, B., and Rosendahl, J. (2018). Efficacy of non-pharmacological interventions for procedural pain relief in adults undergoing burn wound care: a systematic review and meta-analysis of randomized controlled trials. Burns 44, 1709-1720. doi: 10.1016/j.burns.2017.11.019

Schmitt, Y. S., Hoffman, H. G., Blough, D. K., Patterson, D. R., Jensen, M. P., Soltani, M., et al. (2011). A randomized, controlled trial of immersive virtual reality analgesia, during physical therapy for pediatric burns. Burns 37, 61-68. doi: 10.1016/j.burns.2010.07.007

Slepian, P. M., Ankawi, B., Himawan, L. K., and France, C. R. (2016). Development and initial validation of the pain resilience scale. J. Pain 17, 462-472. doi: 10.1016/j.jpain.2015.12.010

Spiegel, B. (2020). VRx: How Virtual Therapeutics Will Revolutionize Medicine. New York, NY: Basic Books.

Stone, A. A., Broderick, J. E., Kaell, A. T., DelesPaul, P. A. E. G., and Porter, L. E. (2000). Does the peak-end phenomenon observed in laboratory pain studies apply to real-world pain in rheumatoid arthritics? J. Pain 1, 212-217. doi: 10.1054/jpai.2000.7568

Sweetman, P. (1999). Anchoring the (postmodern) self? body modification, fashion and identity. Body Soc. 5, 51-76. doi: 10.1177/1357034X99005002004

Tashjian, V. C., Mosadeghi, S., Howard, A. R., Lopez, M., Dupuy, T., Reid, M., et al. (2017). Virtual reality for management of pain in hospitalized patients: results of a controlled trial. JMIR Mental Health. 4:e9. doi: 10.2196/men tal. 7387 
Technalysis Research (2018). VR/AR Headsets Average Session Time in U.S. Statista. Retrieved from: https:/www.statista.com/statistics/831819/us-virtualaugmented-reality-device-average-session-time/

Trost, Z., France, C., Anam, M., and Shum, C. (2021). Virtual reality approaches to pain: toward a state of the science. Pain 162, 325-331.

Uman, L. S., Stewart, S. H., Watt, M. C., and Johnston, A. (2006). Differences in high and low anxiety sensitive women's responses to a laboratory-based cold pressor task. Cogn. Behav. Ther. 35, 189-197. doi: 10.1080/16506070600898512

van den Berg, A. E., Maas, J., Verheij, R. A., and Groenewegen, P. P. (2010). Green space as a buffer between stressful life events and health. Soc. Sci. Med. 70, 1203-1210. doi: 10.1016/j.socscimed.2010.01.002

Weiss, R. (1994). Pediatric pain, predictive inference, and sensitivity analysis. Eval. Rev. 18, 651-677. doi: 10.1177/0193841X9401800601

Wells, N. M., and Evans, G. W. (2003). Nearby nature. Environ. Behav. 35, 311-330. doi: 10.1177/0013916503035003001

Wender, R., Hoffman, H. G., Hunner, H. H., Seibel, E. J., Patterson, D. R., and Sharar, S. R. (2009). Interactivity influences the magnitude of virtual reality analgesia. J. Cyber Ther. Rehabil. 2, 27-33.
Won, A., Bailey, J., Bailenson, J., Tataru, C., Yoon, I., and Golianu, B. (2017). Immersive virtual reality for pediatric pain. Children 4:52. doi: 10.3390/children4070052

Wright, J. L., Hoffman, H. G., and Sweet, R. M. (2005). Virtual reality as an adjunctive pain control during transurethral microwave thermotherapy. Urology. 66:1320. doi: 10.1016/j.urology.2005.06.123

Conflict of Interest: The authors declare that the research was conducted in the absence of any commercial or financial relationships that could be construed as a potential conflict of interest.

Copyright (c) 2021 Pimentel, Kalyanaraman, Fillingim and Halan. This is an openaccess article distributed under the terms of the Creative Commons Attribution License (CC BY). The use, distribution or reproduction in other forums is permitted, provided the original author(s) and the copyright owner(s) are credited and that the original publication in this journal is cited, in accordance with accepted academic practice. No use, distribution or reproduction is permitted which does not comply with these terms. 